Advanced Studies in Theoretical Physics, Vol. 8, No. 18, pp.771-779 (2014).

http://dx.doi.org/10.12988/astp.2014.47101

\title{
The procedure of finding the stress-energy tensor and equations of vector field of any form
}

\author{
Sergey G. Fedosin \\ Sviazeva Str. 22-79, Perm, 614088, Perm region, Russian Federation \\ e-mail $\underline{\text { intelli@list.ru }}$
}

\begin{abstract}
A method allowing us to introduce into the Lagrangian the terms, which characterize an arbitrary vector field of a system, is described. As a result of applying the principle of least action it becomes possible to find all the main characteristics of this field, including its energy and momentum, field equations, force of interaction with the matter.
\end{abstract}

Keywords: four-potential; pressure field; acceleration field; field equations.

\section{Introduction}

The field concept is widely used not only in the gravitation theory but also in other physical theories. Further we will consider the properties of vector fields in fourdimensional space.

The main characteristic of the electromagnetic vector field is the 4-potential $A_{\mu}=\left(\frac{\varphi}{c},-\mathbf{A}\right)$, where $c$ is the speed of light, $\varphi$ and $\mathbf{A}$ denote the scalar and vector potentials, respectively. If the system contains a set of particles, each of which generates its own potential, then the potentials $\varphi$ and $\mathbf{A}$ of the system of particles depend mainly on the general system parameters - the dimensions of the system, the total charge, etc. Besides the system's potentials correspond to the superposition principle the potentials of all the particles. We can determine all the main characteristics of the system's electromagnetic field with the help of the 4-potential.

Thus before we find the 4- potential of the system, we need to determine the 4-potential of a single particle. This can be done as follows: the invariant scalar potential $\varphi_{p}$ of the particle should be divided by the square of the speed of light to get the necessary dimension, and then multiply it by the covariant 4-velocity: 


$$
A_{\mu}=\frac{\varphi_{p}}{c^{2}} u_{\mu}=\left(\frac{\varphi_{p}}{c^{2}} \frac{d x_{0}}{d \tau}, \frac{\varphi_{p}}{c^{2}} \frac{d x_{1}}{d \tau}, \frac{\varphi_{p}}{c^{2}} \frac{d x_{2}}{d \tau}, \frac{\varphi_{p}}{c^{2}} \frac{d x_{3}}{d \tau}\right)=\left(\frac{\varphi}{c},-A_{1},-A_{2},-A_{3}\right)
$$

The potential $\varphi_{p}$ is considered to be invariant, if it is determined in the reference frame $K_{p}$, which is rigidly associated with the particle. We can see in (1), that $\varphi_{p}$ is associated with the scalar potential $\varphi$ and the three components of the vector potential $\mathbf{A}$ of a particle in an arbitrary reference frame $K$, in which the particle has a 4-velocity $u_{\mu}=\frac{d x_{\mu}}{d \tau}$ (where $d x_{\mu}$ is a 4-displacement with the covariant index, $\tau$ is the proper time of the particle). In order to find the 4-potential of the system, it is necessary to integrate (1) over all of the system's particles.

\section{Pressure field}

We will turn now to the pressure field, the properties of which must be taken into account in calculating the metric inside the material bodies, as well as in determining the equation of motion and of the state of matter. The existing definitions of the pressure field and its energy-momentum are derived by means of generalization of the formulas of classical mechanics. For example, in the general theory of relativity (GTR) the following pressure tensor [1] is used for ideal liquid:

$$
P^{\mu v}=\frac{p}{c^{2}} u^{\mu} u^{v}-g^{\mu v} p
$$

In (2) the pressure $p$ represents a scalar field.

The tensor $\phi^{\mu v}=\rho u^{\mu} u^{v}$ is considered to be the matter characteristic in GTR, and the total stress-energy tensor of the matter with pressure $p$ and density $\rho$ is equal to: $T^{\mu v}=\phi^{\mu v}+P^{\mu v}$.

Now we will answer the question whether we can consider the pressure field not just scalar but a four-dimensional vector field? By definition, a vector field at each point is described by a certain vector. In continuously distributed matter the particles are so close to each other, that they constantly interact with each other. In this case, we can assume that the direction of the vector of one particle's pressure on another is parallel to the vector of 
particle velocity. If a vector field of velocity is specified for the particles, then the vector field of pressure can be considered as the consequence of the velocity field.

On the other hand, the pressure, even in the absence of particles' motion when it looks like a scalar field, makes its contribution to the mass-energy of the particles. Since the pressure has meaning both of a scalar and of a three-dimensional vector, there must be a 4vector, where the pressure is part of the scalar and vector components. It is natural to call such a 4-vector a 4-potential of the pressure field.

We will determine the 4-potential of the pressure field similarly to (1):

$$
\pi_{\mu}=\frac{p_{0}}{\rho_{0} c^{2}} u_{\mu}=\left(\frac{\wp}{c},-\mathbf{\Pi}\right),
$$

where $p_{0}$ and $\rho_{0}$ denote the pressure and density in the reference frame $K_{p}$ of the particle, the dimensionless ratio $\frac{p_{0}}{\rho_{0} c^{2}}$ is proportional to the pressure energy of the particle per particle's unit mass, $\wp$ and $\Pi$ are the scalar and vector potentials of the pressure field.

Then applying the 4-rotor we find the antisymmetric pressure tensor $f_{\mu \nu}$, consisting of six components, belonging to two vectors $\mathbf{C}=\left(C_{x}, C_{y}, C_{z}\right), \quad \mathbf{I}=\left(I_{x}, I_{y}, I_{z}\right)$ :

$$
\begin{gathered}
f_{\mu \nu}=\nabla_{\mu} \pi_{\nu}-\nabla_{\nu} \pi_{\mu}=\partial_{\mu} \pi_{\nu}-\partial_{\nu} \pi_{\mu} . \\
f_{\mu \nu}=\left(\begin{array}{cccc}
0 & \frac{C_{x}}{c} & \frac{C_{y}}{c} & \frac{C_{z}}{c} \\
-\frac{C_{x}}{c} & 0 & -I_{z} & I_{y} \\
-\frac{C_{y}}{c} & I_{z} & 0 & -I_{x} \\
-\frac{C_{z}}{c} & -I_{y} & I_{x} & 0
\end{array}\right) .
\end{gathered}
$$

Now, using (4) we can construct a tensor invariant $\frac{c}{16 \pi \sigma} f_{\mu v} f^{\mu \nu}$, where $\sigma$ should be determined. The pressure field equations are obtained from the principle of least action, 
while the sum should be substituted into the Lagrangian: $-\frac{1}{c} \pi_{\mu} J^{\mu}-\frac{c}{16 \pi \sigma} f_{\mu \nu} f^{\mu \nu}$, where $J^{\mu}$ is the mass 4-current. For comparison, all the properties of the electromagnetic field are obtained by varying a similar sum: $-\frac{1}{c} A_{\mu} j^{\mu}-\frac{c \varepsilon_{0}}{4} F_{\mu \nu} F^{\mu v}$, where $j^{\mu}$ is the electromagnetic current, $\varepsilon_{0}$ is the vacuum permittivity, $F_{\mu \nu}$ is the electromagnetic tensor.

One of the results of the Lagrangian variation is the stress-energy tensor of the pressure field [2]:

$$
P^{\alpha \beta}=\frac{c^{2}}{4 \pi \sigma}\left(-g^{\alpha \nu} f_{\kappa \nu} f^{\kappa \beta}+\frac{1}{4} g^{\alpha \beta} f_{\mu \nu} f^{\mu \nu}\right)
$$

This tensor with other fields' tensors is part of the right side of the equation for determining the metric, and the left side of this equation contains the Ricci tensor and scalar curvature. With the help of tensor (4) or tensor (6) we can determine the density of 4-force in the equation of matter motion that arises due to the pressure:

$$
f_{\beta \sigma} J^{\sigma}=-\nabla^{k} P_{\beta k}
$$

We also find the pressure field equations:

$$
\nabla_{v} f^{\mu v}=-\frac{4 \pi \sigma}{c^{2}} J^{\mu}, \quad \quad \varepsilon^{\alpha \beta \gamma \delta} \nabla_{\gamma} f_{\alpha \beta}=0
$$

where $\varepsilon^{\alpha \beta \gamma \delta}$ is the Levi-Civita symbol.

Equations (7) in the limit of the special theory of relativity with regard to (5) look like Maxwell equations:

$$
\nabla \cdot \mathbf{C}=4 \pi \sigma \gamma \rho_{0}, \quad \nabla \times \mathbf{I}=\frac{1}{c^{2}} \frac{\partial \mathbf{C}}{\partial t}+\frac{4 \pi \sigma \gamma \rho_{0} \mathbf{v}}{c^{2}}, \quad \nabla \cdot \mathbf{I}=0, \quad \nabla \times \mathbf{C}=-\frac{\partial \mathbf{I}}{\partial t}
$$

Here $\gamma=\frac{1}{\sqrt{1-v^{2} / c^{2}}}$ is the Lorentz factor, $\mathbf{v}$ is the velocity of a point particle of matter. 
If we substitute (4) in the first equation in (7) in the form: $f^{\mu \nu}=\nabla^{\mu} \pi^{v}-\nabla^{v} \pi^{\mu}$, we obtain:

$$
\nabla_{v} \nabla^{\mu} \pi^{v}-\nabla_{v} \nabla^{v} \pi^{\mu}=-\frac{4 \pi \sigma}{c^{2}} J^{\mu}
$$

In case if 4-potential gauge $\nabla_{v} \pi^{v}=0$ in the left side of the equation we have $\nabla_{v} \nabla^{\mu} \pi^{v}=\pi^{s} R_{s}^{\mu}$, where $R_{s}^{\mu}$ denotes the Ricci tensor with mixed indices. On the other hand, 4-d'Alembertian acting on the 4-vector $\pi^{\mu}$ is determined in the general case as follows:

$$
\nabla_{v} \nabla^{v} \pi^{\mu}=\square \pi^{\mu}=g^{\rho v} \partial_{\rho} \partial_{v} \pi^{\mu}+\pi^{s} R_{s}^{\mu}+g^{\rho v}\left(\Gamma_{v s}^{\mu} \partial_{\rho} \pi^{s}-\Gamma_{\rho v}^{s} \partial_{s} \pi^{\mu}+\Gamma_{s \rho}^{\mu} \partial_{v} \pi^{s}+\pi^{s} \partial_{s} \Gamma_{v \rho}^{\mu}\right) .
$$

As a result the terms with the Ricci tensor are canceled and we have the following:

$$
g^{\rho v} \partial_{\rho} \partial_{v} \pi^{\mu}+g^{\rho v}\left(\Gamma_{v s}^{\mu} \partial_{\rho} \pi^{s}-\Gamma_{\rho v}^{s} \partial_{s} \pi^{\mu}+\Gamma_{s \rho}^{\mu} \partial_{v} \pi^{s}+\pi^{s} \partial_{s} \Gamma_{v \rho}^{\mu}\right)=\frac{4 \pi \sigma}{c^{2}} J^{\mu}
$$

Equation (9) represents the wave equation for the 4-potential of the pressure field, which allows us to find the pressure distribution inside the massive bodies. In particular, for spherical bodies with approximately constant density the pressure decreases from the body center to its surface, due to the presence of the negative term in the formula for the pressure, which is proportional to the square of the current radius. From (9) we can estimate the pressure at the center of the massive body:

$$
p_{c} \approx \frac{3 \sigma M^{2}}{8 \pi R^{4}}
$$

where $\sigma=3 G, G$ is the gravitational constant, $M$ and $R$ denote the body mass and radius.

\section{Acceleration field}


The foregoing describes the procedure of obtaining the stress-energy tensor and the vector field equations of any kind. In particular, the above-mentioned procedure was also applied in [2] in order to find the stress-energy tensor of matter in a covariant way. As the 4potential of the acceleration field the covariant 4- velocity was used without additional factors:

$$
u_{\mu}=\left(\frac{\vartheta}{c},-\mathbf{U}\right)
$$

where $\vartheta$ and $\mathbf{U}$ denote the scalar and vector potentials, respectively.

The tensor of the acceleration field is given by:

$$
\begin{gathered}
u_{\mu \nu}=\nabla_{\mu} u_{v}-\nabla_{\nu} u_{\mu}=\partial_{\mu} u_{v}-\partial_{\nu} u_{\mu} . \\
u_{\mu \nu}=\left(\begin{array}{cccc}
0 & \frac{S_{x}}{c} & \frac{S_{y}}{c} & \frac{S_{z}}{c} \\
-\frac{S_{x}}{c} & 0 & -N_{z} & N_{y} \\
-\frac{S_{y}}{c} & N_{z} & 0 & -N_{x} \\
-\frac{S_{z}}{c} & -N_{y} & N_{x} & 0
\end{array}\right),
\end{gathered}
$$

where the vectors $\mathbf{S}=\left(S_{x}, S_{y}, S_{z}\right)$ and $\mathbf{N}=\left(N_{x}, N_{y}, N_{z}\right)$ define the particle's accelerations.

The contribution of the acceleration field into the Lagrangian is given by the sum: $-\frac{1}{c} u_{\mu} J^{\mu}-\frac{c}{16 \pi \eta} u_{\mu \nu} u^{\mu \nu}$, where $\eta$ is to be determined. The stress-energy tensor of the acceleration field appears as a result of variation:

$$
B^{\alpha \beta}=\frac{c^{2}}{4 \pi \eta}\left(-g^{\alpha \nu} u_{\kappa \nu} u^{\kappa \beta}+\frac{1}{4} g^{\alpha \beta} u_{\mu \nu} u^{\mu \nu}\right)
$$


The 4-acceleration in the equation of motion of a small particle of continuously distributed matter is found either with the help of tensor (11) or tensor (13):

$$
\rho_{0} \frac{D u_{\beta}}{D \tau}=-u_{\beta \sigma} J^{\sigma}=\nabla^{k} B_{\beta k}
$$

Like any vector field, the acceleration field is given by the corresponding equations:

$$
\nabla_{\beta} u^{\alpha \beta}=-\frac{4 \pi \eta}{c^{2}} J^{\alpha}, \quad \varepsilon^{\alpha \beta \gamma \delta} \nabla_{\gamma} u_{\alpha \beta}=0
$$

These equations in the special theory of relativity are the equations for the vectors $\mathbf{S}$ and $\mathbf{N}$ from (12):

$$
\nabla \cdot \mathbf{S}=4 \pi \eta \gamma \rho_{0}, \quad \nabla \times \mathbf{N}=\frac{1}{c^{2}} \frac{\partial \mathbf{S}}{\partial t}+\frac{4 \pi \eta \gamma \rho_{0} \mathbf{v}}{c^{2}}, \quad \nabla \cdot \mathbf{N}=0, \quad \nabla \times \mathbf{S}=-\frac{\partial \mathbf{N}}{\partial t}
$$

The stress-energy tensors of the pressure field $P^{\alpha \beta}(6)$ and the acceleration field $B^{\alpha \beta}$ (13) are constructed in a covariant way using the 4-potentials and in the covariant theory of gravitation they substitute the tensor $P^{\mu v}$ in (2) and the tensor $\phi^{\mu v}=\rho u^{\mu} u^{v}$, respectively.

Similarly to (9) we obtain the wave equation for the velocity field inside the bodies:

$$
g^{\rho v} \partial_{\rho} \partial_{v} u^{\mu}+g^{\rho v}\left(\Gamma_{v s}^{\mu} \partial_{\rho} u^{s}-\Gamma_{\rho v}^{s} \partial_{s} u^{\mu}+\Gamma_{s \rho}^{\mu} \partial_{v} u^{s}+u^{s} \partial_{s} \Gamma_{v \rho}^{\mu}\right)=\frac{4 \pi \eta}{c^{2}} J^{\mu}=\frac{4 \pi \eta \rho_{0}}{c^{2}} u^{\mu}
$$

The solution of this equation allows us to calculate the velocity of the particles' motion inside the spherical body as a function of the current radius. The kinetic energy of the particles depends on their velocity and specifies the kinetic temperature. Consequently, it becomes possible to find the equilibrium temperature distribution inside the massive bodies. In particular, for the temperature at the center we can approximately write the following:

$$
T_{c} \approx \frac{\eta M_{p} M}{3 k R}
$$


where $\eta=3 G, M_{p}$ denote the mass of a typical body particle, usually it is the mass of a hydrogen atom, $k$ is the Boltzmann constant.

\section{Conclusion}

Despite the fact that the formulas (10) and (16) were found in the assumption of uniform density, they are well satisfied for gas clouds, planets and stars. Good agreement is observed for Bok globules, the Earth and neutron star, as well as for the temperature inside the Sun [3]. The difference occurs only for the pressure inside the Sun, where it is 58 times less than in the standard model. This is probably due to the fact that thermonuclear reactions take place inside the Sun, which increase the pressure.

We believe that the massive bodies, held in equilibrium by gravitation force, contain radial gradients of the potentials of gravitation, pressure, particles' kinetic energy and other quantities. These gradients are the essential components that ensure the system's stability at a given matter state. If we assume the validity of the gravitation mechanism in Le Sage's theory [4], then at equilibrium the temperature of the interior of a massive cosmic body cannot fall below the value that is obtained from the virial theorem. Despite the constant emission from the surface of the body, the necessary energy inflow is ensured by gravitons falling on the body. In Le Sage's model the graviton fluxes penetrating the matter not only create the gravitational force, but also leave some part of their energy inside the body, warming it.

Thus, we have introduced a procedure, according to which it is necessary first to determine the scalar potential of an arbitrary vector field, inherent in a single particle. After that by means of standard methods all the characteristics of this field are derived, including the field equations, its stress-energy tensor and the type of the force, exerted by the field on the particles.

We must note that the 4-potential of the field is expressed as a covariant 4-vector, and the matter energy in this field depends on the product of the 4-potential and the mass (electromagnetic) 4-current, taken with the contravariant index. The field tensor has doubly covariant indices as the consequence of the 4-curl acting on the 4-potential. In order to find this tensor with contravariant indices the metric tensor is required. Contraction of the field tensor with itself gives the tensor invariant, which is required in the Lagrangian to carry out variation and to express relationship between the matter, metric and field in the appropriate equations. Another peculiarity of this approach is that the field equations (8) and (14) are similar in form to Maxwell equations. 
The previously described approach was used in [5], [6], [7] to find also the 4-potential of the gravitational field, its stress-energy tensor, gravitational 4-force and field equations in the framework of the Covariant Theory of Gravitation (CTG).

In CTG the gravitational field is divided from the metric field, gravitation becomes an independent field with its own energy, momentum and action in the form of gravitational force. As a result, the metric is only necessary to describe deviations of the results of gravitational experiments from their form in the special theory of relativity. The essential part of the Lagrangian in CTG is the constant $\Lambda$, which is called cosmological constant. With the help of this constant the Hamiltonian gauge is performed so that the system's energy could be determined unambiguously.

\section{References}

1. C. W. Misner, K. S. Thorne, and J. A. Wheeler, Gravitation (W. H. Freeman, San Francisco, CA, 1973).

2. Fedosin S.G. About the cosmological constant, acceleration field, pressure field and energy. vixra.org, 5 Mar 2014.

3. Fedosin S.G. The Integral Energy-Momentum 4-Vector and Analysis of 4/3 Problem Based on the Pressure Field and Acceleration Field. American Journal of Modern Physics. Vol. 3, No. 4, 2014, pp. 152-167. doi: 10.11648/j.ajmp.20140304.12.

4. Fedosin S.G. Model of Gravitational Interaction in the Concept of Gravitons. Journal of Vectorial Relativity, 2009, Vol. 4, No. 1, P.1-24.

5. Fedosin S.G. The Principle of Least Action in Covariant Theory of Gravitation. Hadronic Journal, 2012, Vol. 35, No. 1, P. 35-70.

6. Fedosin S.G. Fizicheskie teorii i beskonechnaia vlozhennost' materii. (Perm, 2009). ISBN 978-5-9901951-1-0.

7. Fedosin S.G. Fizika i filosofiia podobiia ot preonov do metagalaktik. (Perm, 1999). ISBN 5-8131-0012-1. 